\title{
Coeliac disease and granulomatous ileocolitis: an association or chance occurrence?
}

\author{
${ }^{1} \mathrm{CE}$ Johns, ${ }^{1} \mathrm{JH}$ Topping, ${ }^{2} \mathrm{C}$ Bloxham, ${ }^{1} \mathrm{~A}$ Dhar \\ ${ }^{1}$ Department of Gastroenterology; 'Department of Pathology, County Durham \& Darlington NHS Foundation Trust, Co. Durham, UK
}

\begin{abstract}
Enteric granulomatous inflammation can be caused by a number of conditions including Crohn's disease, sarcoidosis, enteric infections, chronic granulomatous disease and also by drug reactions. Granulomas have also been described in microscopic colitis associated with certain medications and autoimmune diseases. The association of granulomatous ileocolitis with coeliac disease is not common. We present a case of coeliac disease with granulomatous ileocolitis with follow-up and repeat histology on a gluten-free diet. We discuss the pathological mechanisms leading to the association of granulomatous ileocolitis with coeliac disease as well as other conditions.
\end{abstract}

Correspondence to A Dhar, Department of Gastroenterology, County Durham \& Darlington NHS Foundation Trust, Cockton Hill Road, Co. Durham, DLI4 6AD, UK

tel. $+44(0) 1388455170$ e-mail anjan.dhar@cddft.nhs.uk

KEYWORDS Microscopic colitis, coeliac disease, granuloma, Crohn's disease

DECLARATION OF INTERESTS This case was presented at the 2008 United European Gastroenterology Week at the annual meeting of the European Gastroenterology Federation. Dr Dhar has received honoraria from pharmaceutical companies for lectures, advisory work and consultancy.

\section{INTRODUCTION}

Chronic diarrhoea is a common reason for referral to gastroenterology services and can be caused by infections, immunologically-mediated diseases such as inflammatory bowel diseases, coeliac disease and microscopic colitis and a functional bowel. Coeliac disease can sometimes occur in association with ulcerative colitis, Crohn's disease or microscopic colitis. Microscopic colitis occurs in two forms, namely lymphocytic colitis and collagenous colitis, which are distinguished by their specific histological appearances. The lamina propria (the tissue layer of the mucous membrane) contains an inflammatory infiltrate in both, but the sub-epithelial collagen band is expanded in collagenous colitis and intra-epithelial lymphocytes are significantly increased in lymphocytic colitis. Microscopic colitis can occur in association with some medications and with autoimmune diseases. Granulomatous inflammation has also been described in this condition. However, the occurrence of granulomatous ileocolitis in coeliac disease is not common and the effect of a glutenfree diet on the course of the ileocolitis has not been reported. We present a case of coeliac disease with granulomatous ileocolitis. The patient was followed up for over a year on a gluten-free diet, monitoring serial quantitative immunoglobulin A ( $\lg \mathrm{A})$ tissue transglutaminase levels. Repeat small bowel and ileocolonic histology were obtained after clinical remission.

\section{CASE REPORT}

A 49-year-old woman presented to gastroenterology services in another hospital in May 2007 with a sixmonth history of watery diarrhoea and tiredness. She thought her diarrhoea worsened after eating fruit but wheat products did not seem to alter her symptoms.
She had not lost weight, had no cutaneous rash or any extra-intestinal manifestations of inflammatory bowel disease (IBD). She had a past medical history of hypertension, which was treated with candesartan, atenolol and bendroflumethiazide. There was no recent history of foreign travel.

Initial investigations revealed negative stool cultures, negative stool Clostridium difficile toxin assay by enzymelinked immunosorbant assay (ELISA), a normal full blood count, serum albumin and C-reactive protein. An antiendomysial antibody test done elsewhere was negative. An ileocolonoscopy was macroscopically normal but biopsies showed evidence of inflammation with numerous small granulomata. These tests indicated intestinal inflammation, and the diagnosis at this time was still being considered as possible early Crohn's disease.

In August 2007 the patient was referred to our hospital for a second evaluation since her symptoms had not resolved and there was a suspicion of Crohn's disease, based on the ileocolonic histology (the presence of granulomas on the histology). On re-evaluation, her symptoms of watery diarrhoea and lethargy were unchanged and non-progressive. She had not lost weight and there was no abdominal pain or rectal bleeding. Once again, her full blood count, albumin and C-reactive protein remained normal. Repeat stool cultures for Yersinia and Campylobacter were negative and a barium small bowel follow-through examination showed no evidence of small bowel disease. Her anti-endomysial antibodies were also tested again and found to be strongly positive this time (an IgA tissue transglutaminase antibody test was not locally available at the time). An oesophago-gastro-duodenoscopy (OGD) was performed and duodenal biopsies taken. An 
ileocolonoscopy was also repeated and the terminal ileum was macroscopically normal (Figure I). The only abnormality in the colon was some erythema at the hepatic flexure (the bend in the colon just under the liver on the right side) (Figure 2). Duodenal histology showed subtotal villous atrophy, increased intra-epithelial lymphocytic infiltrate and enhanced apoptosis consistent with untreated coeliac disease (this was considered to be consistent with Marsh Illb changes, diagnostic of coeliac disease) (Figure 3). Ileocolonic histology revealed granulomatous inflammation of the ileum (Figure 4) and colon (Figure 5). No villous atrophy was reported in the ileal biopsies (indicating that the degree of mucosal damage was mild). In view of a clinical diagnosis of coeliac disease, it was felt that additional small bowel imaging by magnetic resonance (MR) enterography or computed tomography (CT) enteroclysis was inappropriate at this stage without a clinical trial of a gluten-free diet.

The patient started on a gluten-free diet and her symptoms of diarrhoea promptly settled. After 12 months, her quantitative IgA tissue transglutaminase level was below 15 $\mathrm{ng} / \mathrm{ml}$ (indicating good compliance with the gluten-free diet) and repeat duodenal biopsies showed a decrease in lamina propria inflammatory cells, with little evidence of crypt hyperplasia, indicative of small bowel mucosal recovery. The small intestinal mucosa had not recovered completely and this is often the case in patients with coeliac disease. lleocolonoscopy showed no macroscopic mucosal lesions but biopsies continued to show granulomatous inflammation in the submucosa without any crypt distortion. She continued to do well despite the histological changes persisting in the terminal ileal and right colonic biopsies, and there was immunological evidence of good compliance with the gluten-free diet and persistent mucosal recovery. The patient has been on follow-up for over three years without any evidence of the development of Crohn's disease in the small bowel or colon.

\section{DISCUSSION}

This is an unusual presentation of coeliac disease with microscopic ileocolonic inflammation and widespread granulomas. The macroscopic abnormalities seen in her colon were subtle and non-specific. We excluded microscopic colitis caused by medications (specifically non-steroidal anti-inflammatory drugs, lansoprazole, ranitidine, acarbose, aspirin, sertraline or ticlopidine, which have all been reported to cause microscopic lymphocytic colitis).' The patient was not on antibiotics or allopurinol, both of which have been associated with granulomatous microscopic colitis. ${ }^{2}$ Infections that can be associated with microscopic colitis (Yersinia, Campylobacter and Clostrium difficile) were also excluded. ${ }^{3}$ Another potential alternative diagnosis is microscopic colitis in association with coeliac disease, as described by Mulhaupt and Nylin (although granulomatous

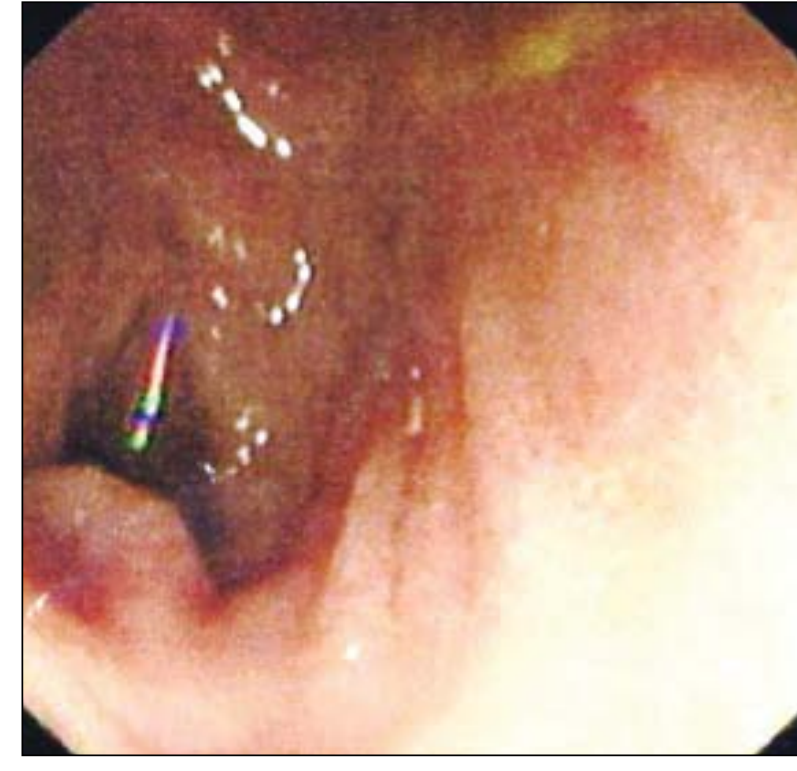

FIGURE I Endoscopic appearance of the terminal ileum.

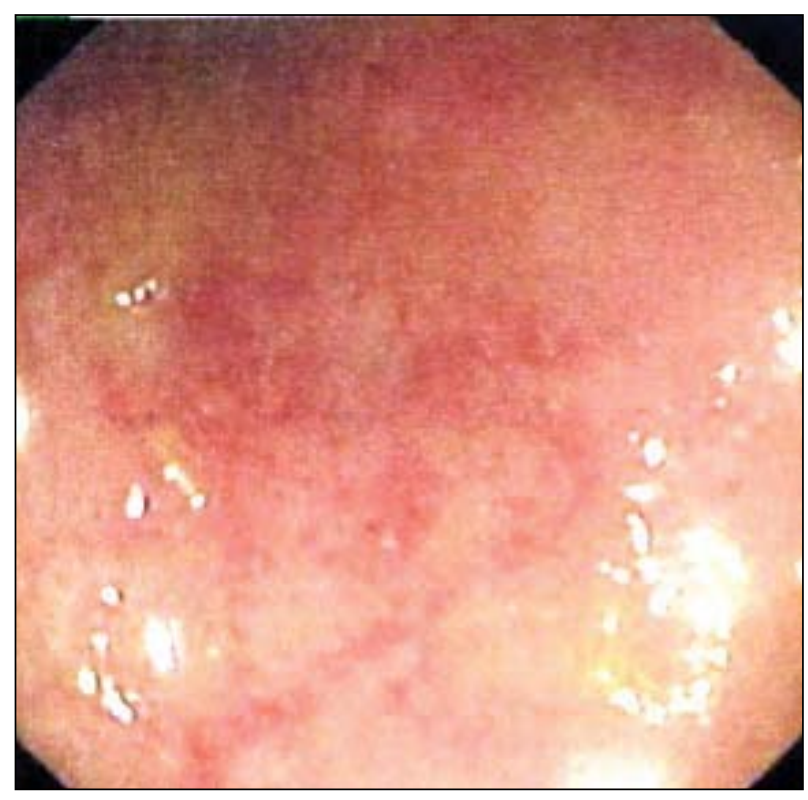

FIGURE 2 Endoscopic appearance of the hepatic flexure.

microscopic colitis is much less common in coeliac disease). ${ }^{3.4}$ Systemic granulomatous diseases such as tuberculosis were excluded by a normal chest radiograph and the absence of specific endoscopic and histological features of intestinal histology. Sarcoidosis was not investigated by serum angiotensin converting enzyme (serum ACE) levels since the patient had a normal chest $\mathrm{X}$-ray and the duodenal histology of villous atrophy made it unethical for further investigations to be carried out without a trial of a gluten-free diet.

The association of Crohn's disease with concomitant coeliac disease is a complex one, particularly with reference to the development of granulomas. One of the 


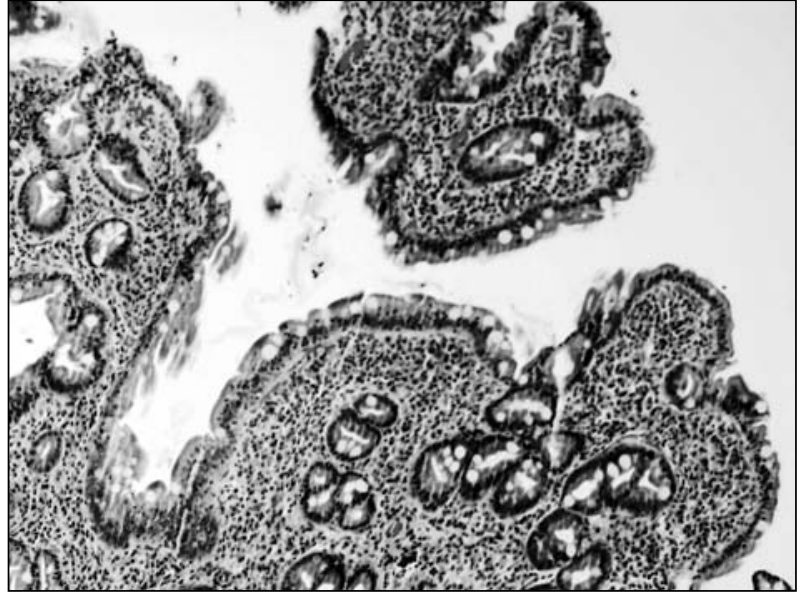

FIGURE 3 Histologic appearance of the duodenum (hematoxylin and eosin [H \& E] stain).

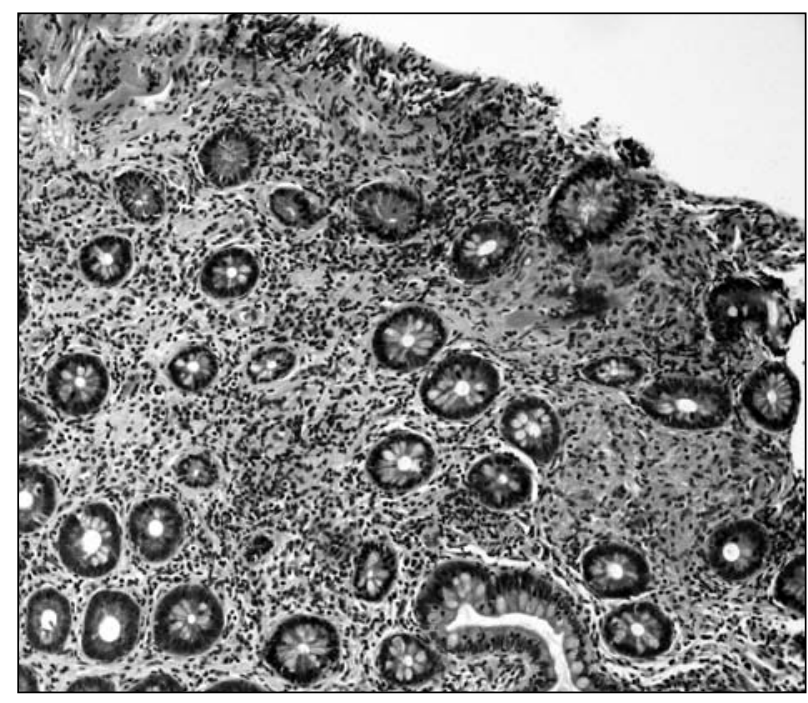

FIGURE 4 Histologic appearance of the ileum (hematoxylin and eosin [H \& E] stain).

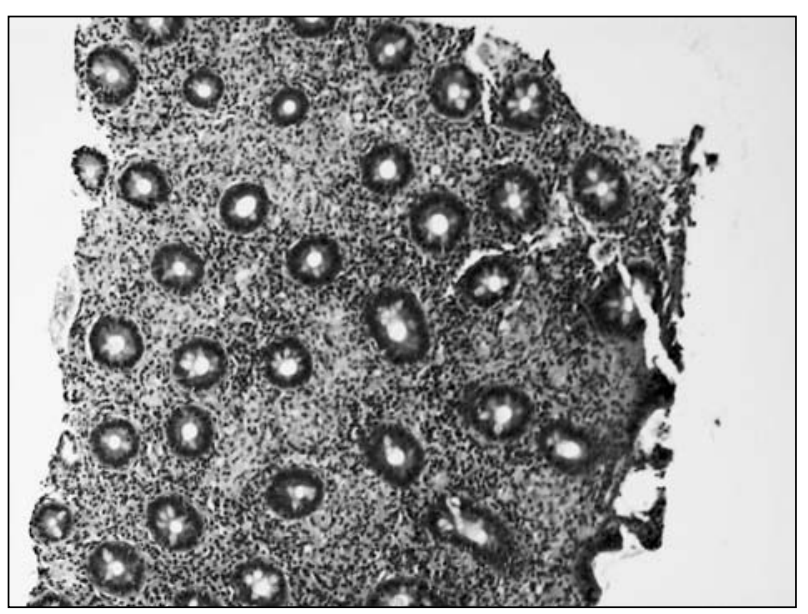

FIGURE 5 Histologic appearance of the colon (hematoxylin and eosin [H \& E] stain). most widely accepted hypotheses for the development of granulomas in Crohn's disease is obstructive lymphangitis, although the antigen that provokes lymphatic endothelial damage is unconfirmed. ${ }^{5}$ Whether gliadin peptide antigens could induce a granulomatous response in the distal small bowel and colon is not known, but they do not evoke such a histological response in the proximal small bowel. We therefore feel that Crohn's ileocolitis was less likely in this patient as there were no other clinical, radiologic, endoscopic or histological features to suggest this diagnosis, and she responded to a gluten-free diet, without any progression of her ileal disease during regular review for over three years.

It is impossible to predict whether this patient will develop Crohn's disease in the future since it is known that patients can have the disease for several years before clinical symptoms present. However, the association of coeliac disease and inflammatory bowel disease has been recognised previously with several examples of shared pathogenic mechanisms. A number of case reports and mortality data indicate an association between coeliac disease and inflammatory bowel disease. ${ }^{6,7}$ The reported prevalence of coeliac disease in patients with Crohn's disease is widely variable, with figures ranging from $<1 \%$ to $25.9 \%$, although it might be argued that this prevalence should be no more than the expected population figure of $1-2 \% .8,9$ One reason for this discrepancy is that positive antigliadin antibiodies and elevated tissue transglutaminase antibodies may occur in Crohn's disease simply as an epiphenomenon, without histological evidence of villous atrophy or indeed, a response to a gluten-free diet. ${ }^{10}$ Tursi et al. report a high incidence of coeliac disease in Crohn's disease but do not report a response to a gluten-free diet." A persuasive pathogenic mechanism is that bacteria with sequences mimicking gliadin cross a damaged, more permeable, intestinal mucosa and provoke an immunological reaction in an already activated immune system. In support of this theory, antibodies to the yeast Saccharomyces cervisiae and other commensal intestinal bacteria are found in the sera from patients with coeliac and Crohn's disease. Specific immunopathological cytokines are also elevated in both diseases." A recent paper describes a genetic association between coeliac disease and inflammatory bowel disease which is mediated by persistent activation of nuclear factokappaB (NF-kB), a key pathway already described for Crohn's disease. ${ }^{2}$ The persistence of mucosal granulomas in the distal small bowel and colon despite good adherence to a gluten-free diet is not surprising - in other granulomatous diseases such as tuberculosis and sarcoidosis, once granulomas have developed, they persist for long periods of time. This appears to be a protective host immune response, where macrophage-driven cellular immune mechanisms target the mucosal antigen and wall it off by the formation of a granuloma. We feel 
that our patient does not have Crohn's disease for several reasons: the benign course of the disease over a long period of time, the normal C-reactive protein and the symptomatic response to a gluten-free diet.

The literature describes several treatments for microscopic colitis: first, medications that could be contributing to the condition should be stopped. Cholestyramine can be effective for concomitant bile salt malabsorption and loperamide is useful to control bowel symptoms. Budesonide and 5 -aminosalicylates ( 5 ASAs) lead to remission of symptoms but there is a high rate of relapse following budesonide cessation. A case of granulomatous microscopic colitis has been reported to respond to prednisolone. ${ }^{13}$ Furthermore, lymphocytic colitis can be self-limiting. A follow-up of 27 patients reported that $93 \%$ were in clinical remission at a mean of 37.8 months. ${ }^{4}$ In contrast, symptoms have persisted for 15 years in some cases of granulomatous microscopic colitis. ${ }^{2}$

\section{CONCLUSION}

This case demonstrates the development of small intestinal granulomatous inflammation in a patient with serological and histologically proven gluten enteropathy, without the clinico-radiological occurrence of Crohn's disease, the most common cause of granulomatous ileocolitis in the Western world. We feel that the possibility of coeliac disease should be considered and excluded whenever the clinical picture of granulomatous ileocolitis is not entirely consistent with Crohn's disease. New insights into the genetics and immunopathogenesis of coeliac disease may provide clues to the widespread intestinal inflammation that may be seen in this condition.

Acknowledgements: This case was discussed at the Challenging Cases in Gastroenterology session at the 2008 United European Gastroenterology week in Vienna and we thank Professor CJJ Mulder for his comments at the presentation. Expert pathological opinion was provided by Professor Neil Shepherd, University of Cranfield, United Kingdom.

\section{REFERENCES}

I Beaugerie L, Pardi DS. Review article: drug induced microscopic colitis - proposal for a scoring system and review of the literature. Aliment Pharmacol Ther 2005; 22:227-84. http://dx.doi.org//0.I I I I/ j.I365-2036.2005.0256I.x

2 Saurine TJ, Brewer JM, Eckstein RP. Microscopic colitis with granulomatous inflammation. Histopathology 2004; 45:82-86. http:// dx.doi.org/I0.IIII/j. I365-2559.2004.01906.x

3 Nhylin N, Bohr J, Eriksson S et al. Microscopic colitis. A common and an easily overlooked cause of chronic diarrhoea. Eur J Intern Med 2008; 19:18I-86. http://dx.doi.org/I0.1016/j.ejim.2008.0I.00 I

4 Mullhaupt B, Guller U, Anabitarte $M$ et al. Lymphocytic colitis: clinical presentation and long term course. Gut 1998; 43:629-33. http://dx.doi.org// 0.1 I36/gut.43.5.629

5 van Kruiningen $\mathrm{HJ}$, Colombel JF. The forgotten role of lymphangitis in Crohn's disease. Gut 2008; 57:1-4. http://dx.doi.org//0.1/36/ gut.2007.123166

6 Peters U,Askling J, Gridley G et al. Causes of death in patients with celiac disease in a population-based Swedish cohort. Arch Intern Med 2003; I63:I566-72. http://dx.doi.org/I0.1001/archinte.163.13.1566

7 Toth E, Ohlsson B, Ljungbery $O$ et al. Celiac disease diagnosed using video capsule endoscopy in a patient with Crohn's disease. Endoscopy 2006; 38;548-49. http://dx.doi.org/I0.1055/s-2006-925342

8 Leeds JS, Horoldt BS, Sidhu R et al. Is there an association between celiac disease and inflammatory bowel diseases? A study of relative prevalence in comparison with population controls. Scand J Gastroenterol 2007; 42:12 I4-20. http://dx.doi.org//0.1080/0036552070/365/ I2

9 Casella G, D'Inca R, Oliva L et al. Prevalence of celiac disease in inflammatory bowel diseases:An IG-IBD multicentre study. Dig Liver Dis 20I0; 42:175-78. http://dx.doi.org//0.1016/j.dld.2009.08.005

10 Di Tola M, Sabbatella L, Anania MC et al. Anti-tissue transglutaminase antibodies in inflammatory bowel disease: new evidence. Clin Chem Lab Med 2004;42; I092-97.http://dx.doi.org/ I0.15 I5/CCLM.2004.225

II Tursi A, Giorgetti GM, Brandimarte G et al. High prevalence of celiac disease among patients affected by Crohn's disease. Inflamm Bowel Dis 2005; I I:662-66. http://dx.doi.org//0.1097/0I.MIB.0000164/95.75207.Ie

I2 Trynka G, Zhernakova A, Romanos J et al. Coeliac disease associated risk variants in TNFAIP3 and REL implicate altered NF-KB signalling. Gut 2009; 58: I078-83. http://dx.doi.org// 0. I |36/ gut.2008.169052

I3 Iwai H, Hisamatsu T, lizuka H et al. Microscopic colitis with granuloma which responded to steroid therapy. Intern Med 2007; 46: I55I-55. http://dx.doi.org/I0.2169/internalmedicine.46.0160 